\title{
Asymptotic Analysis of the Shape and Composition of Alloy Islands in Epitaxial Solid Films
}

\author{
M. Blanariu ${ }^{a}$ and B. J. Spencer ${ }^{b} 1$ \\ ${ }^{a}$ Department of Mathematics, Dalton State College, Dalton, GA 30720, USA \\ ${ }^{b}$ Department of Mathematics, State University of New York at Buffalo, \\ Buffalo, New York 14260-2900, USA
}

\begin{abstract}
We consider the formation of solid drops ("islands") occurring in the growth of strained solid films. Beginning from a detailed model for the growth of an alloy film that incorporates the coupling between composition, elastic stress and the morphology of the free boundary, we develop an asymptotic description of the shape and compositional nonuniformity of small alloy islands grown at small deposition rates. A key feature of the analysis is a "thin domain" scaling in the island which enables recasting the free boundary problem into a set of integrodifferential equations for the shape and composition profile. The integrodifferential system can be decomposed into two parts: one part gives a single integrodifferential equation for the shape analogous to that obtained for a single-component island determined by Shanahan and Spencer (2002), and the other part gives the composition profile in terms of the shape. Thus, the shape of an alloy island is identical to that of a single-component island with the same system parameters, but with a nonuniform composition that depends on the stress-composition coupling and alloy solution thermodynamics. Finally, we characterize the structure and magnitude of the compositional nonuniformity and also interpret our theoretical results in the context of SiGe alloy films.
\end{abstract}

Key words: free boundary problem, alloy film, quantum dot, elasticity, compositional strain AMS subject classification: 74M99, 74G10

\footnotetext{
${ }^{1}$ Corresponding author. E-mail: spencerb@buffalo.edu
} 


\section{Introduction}

Since an important component of many semiconductor devices are strained solid films, it is necessary to understand the processes by which these films are grown. Due to the stored elastic energy in the strained film, the growth of planar strained films is susceptible to a morphological instability $[2,3]$. This morphological instability is usually not desirable from the point of view of growing strained solid films for industrial purposes, because it modifies the electronic structure of the semiconductor devices. However, there has been recent interest in using the instability as a natural mechanism for "self-assembly" of materials with novel features, such as "quantum dots" [6].

Shanahan and Spencer [5] (hereafter referred as SS) analyzed the equilibrium morphology of a strained single-component film for the case where it wets the substrate (Stranski-Krastanow growth). In the Stranski-Krastanow growth mode, the first few monolayers of the film grow layer by layer, forming a "wetting layer." Subsequent growth occurs in the form of mounds or "islands" on the top of the wetting layer. Using an asymptotic analysis based on a "small-slope" approximation, SS reduced the free boundary problem for the island shape to a self-contained integro-differential equation. They found solutions to this equation corresponding to axisymmetric island shapes. In this paper, we extend their analysis to the more general case of a two-component alloy film. This extension is nontrivial because it introduces the composition within the island as an additional unknown.

We use the detailed, realistic model for the strained alloy film growth developed by Spencer, Voorhees, and Tersoff [9] (hereafter referred to as SVT). This model describes the moving boundary of the film surface with underlying alloy composition and elastic strain. In this model, the film surface evolves through the action of surface diffusion and deposition. The coupling between surface morphology, alloy composition and elastic strain plays a critical role in determining the behavior. Our goal in this paper is to understand the behavior of this complicated model for one aspect of the morphology development relevant to film growth; that is, island formation.

We consider the equilibrium solutions of our model for thin films by determining asymptotic solutions for the island shape and composition profile. We use a small length scale in the depth direction to solve asymptotically the elasticity problem in the film. This procedure gives us two coupled surface equations for the island shape and composition profile. We find that our results for the shape can be directly related to the results for single-component films, and we find a generic "map" of the composition nonuniformity in small alloy islands. We present our findings in terms of material parameters for SiGe films. These results were announced in [8]; here we present the complete model formulation and the details of the asymptotic analysis.

The rest of the paper is organized as follows. In Section 2., we describe our mathematical model and nondimensionalize the system. In Section 3., we seek to determine asymptotic solutions for thin islands. In Section 4., we describe the results for axisymmetric islands. In Section 5., we recover the results obtained for the single-component film. In Section 6., we present the results for alloy films. In Section 7., we compare our theoretical results for $\mathrm{Si}_{1-X} \mathrm{Ge}_{X}$ films on $\mathrm{Si}_{1-Y} \mathrm{Ge}_{Y}$ substrates to recent experiments. In Section 8., we present our conclusions. 


\section{Mathematical Model}

\subsection{Governing equations}

We begin with the equilibrium case of the SVT model for an alloy film [9]. The formation of islands requires a model for the wetting interaction of the film and substrate, which is not in SVT. To describe this interaction, we use the wetting layer model employed by SS, in which a wetting layer of negligible thickness covers the substrate, and such that the energy required to remove the wetting layer from the substrate is large relative to the energies involved in the formation of the islands. Therefore, we consider the wetting layer as being a layer of the film of vanishing thickness which is in effect "glued" to the substrate.

Our goal is to determine the shape and the composition profile of an equilibrium island. This problem is more complicated than the single-component case because for the single-component case, we only need to find the shape of a given amount of material. For the alloy case, we need to find the shape and also the composition profile. The composition profile in the island is formed by a two-step process. First, as material is deposited on the island surface, the alloy components can segregate by surface diffusion along the surface in response to the nonuniform strain state. Then, as new material is deposited on top of the existing surface layer, the old surface is buried and becomes part of the "bulk." Since diffusion within the bulk is typically negligible, the buried surface segregation profiles create the composition pattern throughout the depth of the island. Thus, to determine a given equilibrium shape and composition, we need the entire history of how the island grew. We consider here the process of slow growth, envisioning the island starting from zero volume, with material added incrementally and rearranging in the surface layer to achieve surface equilibrium. As the island grows in this quasi-static way, the history of surface compositions is buried to give the the composition depth profile. The more general problem involves faster growth, the dynamics of surface diffusion, and would account for surfaces which were buried before reaching equilibrium.

The formulation of the model is as follows. We seek to determine the equilibrium island shapes $z=h(x, y)$ with composition profiles $C_{B}(x, y, z)$. We describe a single isolated island and thus seek solutions for which $h(x, y)>0$ within a region $\mathcal{D}$ within a closed boundary with $h=0$ on the island boundary and outside the boundary. The surface equations for equilibrium are given by

$$
\begin{gathered}
\mu_{A}=\mu_{A}^{0}\left(C_{A}\right)+\Omega_{\mathrm{vol}}\left[\frac{1}{2} T_{k l}^{F}\left(E_{k l}^{F}-E_{k l}^{c}\right)+\gamma \kappa+C_{B} \eta_{0} T_{k k}^{F}\right] \quad \text { on } z=h \text { in } \mathcal{D} \\
\mu_{B}=\mu_{B}^{0}\left(C_{B}\right)+\Omega_{\mathrm{vol}}\left[\frac{1}{2} T_{k l}^{F}\left(E_{k l}^{F}-E_{k l}^{c}\right)+\gamma \kappa-C_{A} \eta_{0} T_{k k}^{F}\right] \quad \text { on } z=h \text { in } \mathcal{D},
\end{gathered}
$$

where

$$
\mu_{i}^{0}\left(C_{i}\right)=G_{i}+k T \ln \left(C_{i}\right)+\Omega_{\text {int }}\left(1-C_{i}\right)^{2} .
$$

Eqs. (2.1)-(2.2) define the chemical potentials of A and B atoms at the surface of the island, where $C_{i}$ is the composition of component $i$ (atomic fraction), $\mu_{i}^{0}\left(C_{i}\right)$ is the chemical potential of component $i$ at composition $C_{i}$ and at the reference pressure, $\Omega_{\mathrm{vol}}$ is the atomic volume of the film, 
$T_{k l}^{F}$ is the stress tensor in the film, $E_{k l}^{F}$ is the strain tensor in the film, $E_{k l}^{c}$ is the compositional strain tensor, $\gamma$ is the surface free energy, $\kappa$ is the surface curvature, $\eta_{0}$ is the solute expansion coefficient measuring the relative size difference of $\mathrm{B}$ atoms and $\mathrm{A}$ atoms, and $h$ is the island shape. Eq. (2.3) specifies that we use a regular solution model, where $G_{i}$ is the molar (per atom) Gibbs free energy at zero pressure of pure component $i, k$ is the Boltzmann constant, $T$ is the absolute temperature, and $\Omega_{\text {int }}$ is the regular solution interaction parameter. In the above, $C_{A}$ is directly determined from $C_{B}$ by $C_{A}+C_{B}=1$. Equilibrium island shapes are solutions to Eqs. (2.1)-(2.2), corresponding to constant chemical potentials $\mu_{A}$ and $\mu_{B}$. In addition to depending on the island shape and surface compositions, the chemical potentials also depend on the elastic strain in the island, which is determined from simultaneously solving the shape and composition-dependent elasticity problem.

The linear elasticity problem in the film/substrate system is given by the following equations. We use isotropic linear elasticity in each of the film and substrate, where compositional stresses are permitted in the film, but the substrate is assumed to be of known, fixed composition. To simplify our analysis, we also assume that the elastic constants in the film and substrate are the same and independent of composition. The stresses and strains are thus given by

$$
T_{i j}=\frac{E}{1+\nu}\left[\left(E_{i j}-E_{i j}^{c}\right)+\frac{\nu}{1-2 \nu}\left(E_{k k}-E_{k k}^{c}\right) \delta_{i j}\right],
$$

where

$$
\begin{aligned}
E_{i j} & =(1 / 2)\left(u_{i, j}+u_{j, i}\right), \\
E_{i j}^{c} & =\eta_{0}\left(C_{B}-C_{B}^{0}\right) \delta_{i j},
\end{aligned}
$$

and where $E$ is Young's modulus, $\nu$ is Poisson's ratio, and the solute expansion coefficient $\eta_{0}$ quantifies the local strain due to deviations from the reference composition $C_{B}^{0}$. The compositional strain is explicitly zero in the substrate because $C_{B}=C_{B}^{0}$ in the substrate. Mechanical equilibrium inside the film and substrate require that the stress tensors in the film and substrate be divergence free,

$$
\begin{gathered}
(1-2 \nu) u_{i, k k}^{F}+u_{k, k i}^{F}-2(1+\nu) \eta_{0} C_{B, i}=0 \quad \text { in } 0<z<h \\
(1-2 \nu) u_{i, k k}^{S}+u_{k, k i}^{S}=0 \quad \text { in } z<0,
\end{gathered}
$$

where $\underline{u}^{F}$ and $\underline{u}^{S}$ denote the displacements vectors in the film and substrate, comma notation denotes differentiation, and repeated indices denote implicit summation. The boundary conditions for the elasticity problem are given by the following equations. The film surface is traction free,

$$
T_{i j}^{F} n_{j}=0 \quad \text { on } z=h,
$$

where $n_{j}$ is the $j$ component of the outward unit normal to the film surface. There is a force balance at the film/surface interface,

$$
T_{3 j}^{F}=T_{3 j}^{S} \quad \text { on } z=0
$$

There is a jump condition on the displacement vectors $\underline{u}^{F}$ and $\underline{u}^{S}$ at the film/substrate interface due to the difference in lattice parameters in the film and substrate,

$$
\underline{u}^{F}=\underline{u}^{S}+\left[\begin{array}{c}
\epsilon_{0} x \\
\epsilon_{0} y \\
0
\end{array}\right] \quad \text { on } z=0
$$


where $\epsilon_{0}$ is the biaxial misfit strain of the film with the substrate. Finally, the displacements in the substrate decay to zero far away,

$$
\underline{u}^{S} \rightarrow 0 \quad \text { as } z \rightarrow-\infty .
$$

Our aim in this paper is to determine the equilibrium island shapes corresponding to slow deposition of alloy of composition $C_{B}^{0}$. The unknowns are the composition $C_{B}(x, y, z)$, displacement vectors $u_{i}^{F}(x, y, z)$ and $u_{i}^{S}(x, y, z)$, and island shape $h(x, y)$. We parameterize the island shapes by their volume $V$, that is

$$
V=\int_{\mathcal{D}} h \mathrm{~d} A
$$

Then, conservation of $\mathrm{B}$ atoms in the island gives

$$
\int_{V} C_{B} \mathrm{~d} V=C_{B}^{0} V
$$

We now set up the scalings to determine the shape of such islands.

\subsection{Scalings and Nondimensional Model}

We nondimensionalize the system by using a characteristic length scale of $l=\gamma / \sigma \epsilon_{0}$, where $\sigma=\epsilon_{0} E /(1-\nu)$ is the biaxial misfit stress. Eqs. (2.1)-(2.14) then become

$$
\begin{gathered}
\mu_{A}=\mu_{A}^{0}\left(C_{A}\right)+\frac{1}{2} T_{k l}^{F}\left(E_{k l}^{F}-E_{k l}^{c}\right)+\kappa+C_{B} \frac{\eta_{0}}{\epsilon_{0}} T_{k k}^{F} \quad \text { on } z=h \text { in } \mathcal{D} \\
\mu_{B}=\mu_{B}^{0}\left(C_{B}\right)+\frac{1}{2} T_{k l}^{F}\left(E_{k l}^{F}-E_{k l}^{c}\right)+\kappa-C_{A} \frac{\eta_{0}}{\epsilon_{0}} T_{k k}^{F} \quad \text { on } z=h \text { in } \mathcal{D} \\
\mu_{i}^{0}\left(C_{i}\right)=G_{i}+T \ln \left(C_{i}\right)+\Omega\left(1-C_{i}\right)^{2} \\
(1-2 \nu) u_{i, k k}^{F}+u_{k, k i}^{F}-\frac{2(1+\nu) \eta_{0}}{\epsilon_{0}} C_{B, i}=0 \quad \text { in } 0<z<h \\
(1-2 \nu) u_{i, k k}^{S}+u_{k, k i}^{S}=0 \quad \text { in } z<0 \\
T_{i j}^{F} n_{j}=0 \quad \text { on } z=h \\
T_{3 j}^{F}=T_{3 j}^{S} \quad \text { on } z=0 \\
\underline{u}^{F}=\underline{u}^{S}+\left[\begin{array}{c}
x \\
y \\
0
\end{array}\right] \quad \text { on } z=0 \\
\underline{u}_{i j}^{S} \rightarrow 0 \quad \text { as } z \rightarrow-\infty \\
\frac{1-\nu}{1+\nu}\left[\begin{array}{l}
\left.\left(E_{i j}-E_{i j}^{c}\right)+\frac{\nu}{1-2 \nu}\left(E_{k k}-E_{k k}^{c}\right) \delta_{i j}\right]
\end{array}\right.
\end{gathered}
$$




$$
\begin{gathered}
E_{i j}=(1 / 2)\left(u_{i, j}+u_{j, i}\right) \\
E_{i j}^{c}=\eta_{0}\left(C_{B}-C_{B}^{0}\right) \delta_{i j} \\
V=\int_{\mathcal{D}} h \mathrm{~d} A \\
\int_{V} C_{B} \mathrm{~d} V=C_{B}^{0} V,
\end{gathered}
$$

where $V$ now denotes the nondimensional volume of the island.

\section{Asymptotic Solutions for Thin Islands}

\subsection{Thin island scalings and expansions}

We describe a small "flat" island, that is, an island whose height is assumed to be much smaller than the island width. We define a small parameter $\delta=V$ measuring the precise volume of the thin island. For a single-component island, SS showed that the island volume has the same scaling as the island height. We introduce the scaled island shape $H(x, y)=h(x, y) / \delta$ and the small space scale in the depth direction $z=\delta Z$ in the film only. We then express the unknowns in the film in terms of the rescaled $Z$ coordinate as

$$
\left[\begin{array}{l}
C_{B}(x, y, z) \\
\underline{u}^{F}(x, y, z)
\end{array}\right]=\left[\begin{array}{c}
\hat{C}_{B}(x, y, Z) \\
\underline{\hat{u}}^{F}(x, y, Z)
\end{array}\right],
$$

and similarly for quantities deriving from $C_{B}$ and $\underline{u}^{F}$. In what follows we drop the hat notation.

We substitute the above scalings into the nondimensional equations. Eqs. (2.15)-(2.16) and Eq. (2.27) become

$$
\begin{gathered}
\mu_{A}=\mu_{A}^{0}\left(C_{A}\right)+\frac{1}{2} T_{k l}^{F}\left(E_{k l}^{F}-E_{k l}^{c}\right)+\kappa+C_{B} \frac{\eta_{0}}{\epsilon_{0}} T_{k k}^{F} \quad \text { on } Z=H \text { in } \mathcal{D} \\
\mu_{B}=\mu_{B}^{0}\left(C_{B}\right)+\frac{1}{2} T_{k l}^{F}\left(E_{k l}^{F}-E_{k l}^{c}\right)+\kappa-C_{A} \frac{\eta_{0}}{\epsilon_{0}} T_{k k}^{F} \quad \text { on } Z=H \text { in } \mathcal{D} \\
\int_{\mathcal{D}} H \mathrm{~d} A=1,
\end{gathered}
$$

where $\kappa=\delta \hat{\kappa}$. The other equations are transformed similarly.

We consider the following expansions for the scaled island shape $H$, the film composition $C_{B}$, the displacement vectors $\underline{u}^{F}$ and $\underline{u}^{S}$, and chemical potentials $\mu_{A}$ and $\mu_{B}$.

$$
\begin{aligned}
H(x, y) & =H_{0}(x, y)+\delta H_{1}(x, y)+\delta^{2} H_{2}(x, y)+\ldots \\
C_{B} & =C_{B}^{0}+\delta C_{B}^{1}(x, y, Z)+\delta^{2} C_{B}^{2}(x, y, Z)+\ldots \\
u_{i}^{F} & =u_{i 0}^{F}(x, y, Z)+\delta u_{i 1}^{F}(x, y, Z)+\delta^{2} u_{i 2}^{F}(x, y, Z)+\ldots \\
u_{i}^{S} & =u_{i 0}^{S}(x, y, z)+\delta u_{i 1}^{S}(x, y, z)+\delta^{2} u_{i 2}^{S}(x, y, z)+\ldots \\
\mu_{A} & =\mu_{A}^{0}+\delta \mu_{A}^{1}+\delta^{2} \mu_{A}^{2}+\ldots \\
\mu_{B} & =\mu_{B}^{0}+\delta \mu_{B}^{1}+\delta^{2} \mu_{B}^{2}+\ldots
\end{aligned}
$$


and similarly expansions for quantities deriving from $H, C_{B}, \underline{u}^{F}$, and $\underline{u}^{S}$. For example, the strain $E_{33}^{F}$ in the film is given by

$$
E_{33}^{F}=\frac{1}{\delta} u_{30, Z}^{F}+u_{31, Z}^{F}+\delta u_{32, Z}^{F}+\delta^{2} u_{33, Z}^{F}+\ldots
$$

We substitute the expansions into the governing equations and collect like terms by order in $\delta$.

\subsection{Problem and results at $\mathbf{O}(1)$}

At $\mathrm{O}(1)$, the elasticity problem is

$$
\begin{array}{rll}
u_{i 0, Z Z}^{F}=0 & \text { in } 0<Z<H_{0} \\
(1-2 \nu) u_{i 0, k k}^{S}+u_{k 0, k i}^{S}=0 & \text { in } z<0
\end{array}
$$

with boundary conditions

$$
\begin{aligned}
T_{3 j}^{F 0} & =0 \quad \text { on } Z=H_{0}, \\
\left.T_{3 j}^{S 0}\right|_{z=0} & =\left.T_{3 j}^{F 0}\right|_{Z=0} \\
\left.\underline{u}_{0}^{S}\right|_{z=0} & =\left.\underline{u}_{0}^{F}\right|_{Z=0}-\left[\begin{array}{c}
x \\
y \\
0
\end{array}\right] \\
\underline{u}_{0}^{S} & \rightarrow 0 \quad \text { as } z \rightarrow-\infty .
\end{aligned}
$$

Since we are interested in determining the shape of a small island, we expect that the leadingorder elasticity solution corresponds to an undeformed substrate and a uniformly strained island with strains corresponding to a planar film,

$$
\underline{u}_{0}^{S}=\left[\begin{array}{l}
0 \\
0 \\
0
\end{array}\right]
$$

and

$$
E_{i j}^{F 0}=\left[\begin{array}{ccc}
1 & 0 & 0 \\
0 & 1 & 0 \\
0 & 0 & -\frac{2 \nu}{1-\nu}
\end{array}\right]
$$

with $\mathrm{O}(1 / \delta)$ terms in $E_{i j}^{F}$ identically zero. Thus, the $\mathrm{O}(1)$ displacements in the film are given by

$$
\underline{u}_{0}^{F}=\left[\begin{array}{l}
x \\
y \\
0
\end{array}\right]
$$


corresponding to a film with uniform misfit on an undeformed substrate. These solutions satisfy Eqs. (3.12)-(3.17), with Eq. (3.14) giving conditions on the $\mathrm{O}(\delta)$ displacements to be used at the next order:

$$
\begin{aligned}
& \left.u_{11, Z}^{F}\right|_{Z=0}=0 \\
& \left.u_{21, Z}^{F}\right|_{Z=0}=0 \\
& \left.u_{31, Z}^{F}\right|_{Z=0}=-2 \nu /(1-\nu) .
\end{aligned}
$$

At $\mathrm{O}(1)$, the chemical potentials are obtained from

$$
\begin{aligned}
& \mu_{A}^{0}=\mu_{A}^{0}\left(C_{A}^{0}\right)+\frac{1}{2} T_{i j}^{F 0} E_{i j}^{F 0}+\frac{\eta_{0}}{\epsilon_{0}} C_{B}^{0} T_{k k}^{F 0} \\
& \mu_{B}^{0}=\mu_{B}^{0}\left(C_{B}^{0}\right)+\frac{1}{2} T_{i j}^{F 0} E_{i j}^{F 0}-\frac{\eta_{0}}{\epsilon_{0}} C_{A}^{0} T_{k k}^{F 0} .
\end{aligned}
$$

Since all leading-order quantities here are constants, the leading-order chemical potentials are also constants. The leading-order shape $H_{0}$ is not determined at $\mathrm{O}(1)$.

\subsection{Problem and results at $\mathbf{O}(\delta)$}

At $\mathrm{O}(\delta)$, the elasticity problem is

$$
\begin{array}{rll}
u_{i 1, Z Z}^{F}=0 & \text { in } 0<Z<H_{0} \\
(1-2 \nu) u_{i 1, k k}^{S}+u_{k 1, k i}^{S}=0 & \text { in } z<0
\end{array}
$$

with boundary conditions

$$
\begin{aligned}
T_{31}^{F 1} & =H_{0, x} \quad \text { on } Z=H_{0} \\
T_{32}^{F 1} & =H_{0, y} \quad \text { on } Z=H_{0} \\
T_{33}^{F 1} & =0 \quad \text { on } Z=H_{0}, \\
\left.T_{3 j}^{S 1}\right|_{z=0} & =\left.T_{3 j}^{F 1}\right|_{Z=0} \\
\left.u_{i 1}^{S}\right|_{z=0} & =\left.u_{i 1}^{F}\right|_{Z=0} \\
\underline{u}_{1}^{S} & \rightarrow 0 \quad \text { as } z \rightarrow-\infty,
\end{aligned}
$$

Eq. (3.26) admits the general solution

$$
u_{i 1}^{F}=A_{i 1}(x, y)+B_{i 1}(x, y) Z .
$$

Imposing the boundary condition Eq. (3.32), we deduce that

$$
A_{i 1}(x, y)=u_{i 1}^{S}(x, y, 0) \text {. }
$$


Substituting Eq. (3.34) into Eqs. (3.22)-(3.23), we obtain

$$
B_{11}(x, y)=B_{21}(x, y)=0 \quad \text { and } \quad B_{31}(x, y)=-\frac{2 \nu}{1-\nu} .
$$

Thus, the $\mathrm{O}(\delta)$ solution to the elasticity problem in the film depends on the $\mathrm{O}(\delta)$ solution to the elasticity problem in the substrate through

$$
\underline{u}_{1}^{F}(x, y, Z)=\left[\begin{array}{c}
u_{11}^{S}(x, y, 0) \\
u_{21}^{S}(x, y, 0) \\
u_{31}^{S}(x, y, 0)-\frac{2 \nu}{1-\nu} Z
\end{array}\right]
$$

To complete the $\mathrm{O}(\delta)$ description of the elastic strains, we need to consider the elasticity problem in the film at $\mathrm{O}\left(\delta^{2}\right)$, since the strain term $E_{33}^{F 1}$ (see Eq. (3.11)) involves the $\mathrm{O}\left(\delta^{2}\right)$ displacement component $u_{32}^{F}$. At $\mathrm{O}\left(\delta^{2}\right)$, the elasticity problem for this component is

$$
u_{32, Z Z}^{F}=\frac{(1+\nu) \eta}{1-\nu} C_{B, Z}^{1} \quad \text { in } 0<Z<H_{0}
$$

which has the solution

$$
u_{32}^{F}=A_{32}(x, y)+B_{32}(x, y) Z+\int_{0}^{Z} \frac{(1+\nu) \eta}{1-\nu} C_{B}^{1} d Z
$$

Using the stress boundary condition $T_{33}^{F 1}=0$ on $Z=H_{0}$ we determine

$$
E_{33}^{F 1}=-\frac{\nu}{1-\nu}\left(u_{11}^{S}(x, y, 0)+u_{22}^{S}(x, y, 0)\right)+\frac{(1+\nu) \eta}{1-\nu} C_{B}^{1}
$$

and hence $E_{33}^{F 1}$ is determined in terms of the $\mathrm{O}(\delta)$ elasticity solution in the substrate and the composition profile in the island.

In summary, the elastic solution in the film has been expressed in terms of the functions $u_{i 1}^{S}(x, y, 0)$, which correspond to the surface displacements in the substrate Eq. (3.35) that give compatible surface tractions in Eq. (3.31). Since $T_{3 j}^{F 1}$ is independent of $Z$, from Eqs. (3.28)-(3.30) and Eqs. (3.31)-(3.33), the $\mathrm{O}(\delta)$ boundary conditions on the substrate are effectively

$$
\begin{aligned}
T_{31}^{S 1} & =H_{0, x} \quad \text { on } z=0 \\
T_{32}^{S 1} & =H_{0, y} \quad \text { on } z=0 \\
T_{33}^{S 1} & =0 \quad \text { on } z=0 \\
\underline{u}_{1}^{S} & \rightarrow 0 \quad \text { as } z \rightarrow-\infty .
\end{aligned}
$$

Thus, the $\mathrm{O}(\delta)$ substrate deformations can be found from response of a semi-infinite solid to prescribed surface tractions depending on $H_{0}$. Once the substrate elastic response is determined, the $\mathrm{O}(\delta)$ stresses and strains in the film can be found from Eq. (3.37). We present the solution of this semi-infinite elasticity problem in terms of the free boundary shape $H_{0}$ in Section 4 . The shape of 
the island $H_{0}$ needs to be determined simultaneously by the chemical potential conditions, which at $\mathrm{O}(\delta)$ are

$$
\begin{aligned}
& \mu_{A}^{1}=C_{B}^{1}\left[\frac{\eta_{0}}{\epsilon_{0}} T_{k k}^{F 0}-\frac{\partial \mu_{A}^{0}}{\partial C_{A}}\left(C_{A}^{0}\right)\right]+E_{i j}^{F 0} T_{i j}^{F 1}+\kappa_{0}+C_{B}^{0} \frac{\eta_{0}}{\epsilon_{0}} T_{k k}^{F 1} \text { on } Z=H_{0} \\
& \mu_{B}^{1}=C_{B}^{1}\left[\frac{\eta_{0}}{\epsilon_{0}} T_{k k}^{F 0}+\frac{\partial \mu_{B}^{0}}{\partial C_{B}}\left(C_{B}^{0}\right)\right]+E_{i j}^{F 0} T_{i j}^{F 1}+\kappa_{0}-C_{A}^{0} \frac{\eta_{0}}{\epsilon_{0}} T_{k k}^{F 1} \text { on } Z=H_{0} .
\end{aligned}
$$

The goal, then, is to find $H_{0}, C_{B}^{1}$, and the corresponding stresses $T_{i j}^{F 1}$ that satisfy Eq. (3.45)-(3.46).

\section{Axisymmetric Islands}

We seek to determine a simple island morphology described by these equations, that of an axisymmetric "bump" as in the work of SS for the single-component films [5]. We describe the island shape in cylindrical coordinates with radial symmetry,

$$
Z=H(r), \quad 0 \leq r \leq R
$$

with

$$
\begin{aligned}
& H^{\prime}(0)=0 \\
& H^{\prime}(R)=0 \\
& H(R)=0,
\end{aligned}
$$

where $R$ is the radius describing the island size, to be determined as part of the solution to the problem. As in SS, Eq. (4.3) represents the zero contact angle condition which arises since the surface energy of the island and the surface energy of the wetting layer are assumed to be the same [7]. In addition to the expansions introduced in Section 3.1., we consider

$$
R=R_{0}+\delta R_{1}+\delta^{2} R_{2}+\ldots
$$

The chemical potentials at $\mathrm{O}(\delta)$ then become

$$
\begin{aligned}
\mu_{A}^{1}=C_{B}^{1}\left[\frac{\eta_{0}}{\epsilon_{0}} T_{k k}^{F 0}-\frac{\partial \mu_{A}^{0}}{\partial C_{A}}\left(C_{A}^{0}\right)\right]+E_{i j}^{F 0} T_{i j}^{F 1}-\frac{1}{r} \frac{d}{d r}\left(r \frac{d H_{0}}{d r}\right)+C_{B}^{0} \frac{\eta_{0}}{\epsilon_{0}} T_{k k}^{F 1} \\
\quad \text { on } Z=H_{0} \text { in } 0 \leq r \leq R_{0} \\
\mu_{B}^{1}=C_{B}^{1}\left[\frac{\eta_{0}}{\epsilon_{0}} T_{k k}^{F 0}+\frac{\partial \mu_{B}^{0}}{\partial C_{B}}\left(C_{B}^{0}\right)\right]+E_{i j}^{F 0} T_{i j}^{F 1}-\frac{1}{r} \frac{d}{d r}\left(r \frac{d H_{0}}{d r}\right)-C_{A}^{0} \frac{\eta_{0}}{\epsilon_{0}} T_{k k}^{F 1} \\
\text { on } Z=H_{0} \text { in } 0 \leq r \leq R_{0} .
\end{aligned}
$$

Note that in Eqs. (4.6)-(4.7), the term $\left.E_{i j}^{F 0} T_{i j}^{F 1}\right|_{Z=H_{0}}=\left.T_{k k}^{F 1}\right|_{Z=H_{0}}$ (see Eq. (3.19) and Eq. (3.30)), thus, in order to determine the island shape and the composition profile, we first need to determine 
the stress term in the film $\left.T_{k k}^{F 1}\right|_{Z=H_{0}}$. Using Eq. (3.37) and Eq. (3.39), we deduce that the strain term in the film $E_{k k}^{F 1}$ is related to the strain term in the substrate $E_{k k}^{S 1}$ and the composition $C_{B}^{1}$ by

$$
E_{k k}^{F 1}=\left.E_{k k}^{S 1}\right|_{z=0}+\frac{(1+\nu) \eta_{0}}{(1-\nu) \epsilon_{0}} C_{B}^{1}
$$

It follows that the stress term in the film $T_{k k}^{F 1}$ is related to the stress term in the substrate $T_{k k}^{S 1}$ and the composition $C_{B}^{1}$ by

$$
T_{k k}^{F 1}=\left.T_{k k}^{S 1}\right|_{z=0}-\frac{2 \eta_{0}}{\epsilon_{0}} C_{B}^{1}
$$

where

$$
\left.T_{k k}^{S 1}\right|_{z=0}=\partial_{x} A_{11}+\partial_{y} A_{21}
$$

Thus, explicitly defining the composition on the island surface as

$$
C_{B}^{S 1}(r)=\left.C_{B}^{1}\right|_{Z=H_{0}}
$$

we obtain a direct relation between the stress at the surface and the surface composition

$$
\left.T_{k k}^{F 1}\right|_{Z=H_{0}}=\left.T_{k k}^{S 1}\right|_{z=0}-\frac{2 \eta_{0}}{\epsilon_{0}} C_{B}^{S 1}
$$

Substituting Eq. (4.11) and Eq. (4.12) into Eqs. (4.6)-(4.7), we obtain the reduced problem

$$
\begin{array}{ll}
\mu_{A}^{1}=a C_{B}^{S 1}-\frac{1}{r} \frac{d}{d r}\left(r \frac{d H_{0}}{d r}\right)+\left.c T_{k k}^{S 1}\right|_{z=0} & \text { for } 0 \leq r \leq R_{0} \\
\mu_{B}^{1}=b C_{B}^{S 1}-\frac{1}{r} \frac{d}{d r}\left(r \frac{d H_{0}}{d r}\right)+\left.d T_{k k}^{S 1}\right|_{z=0} \quad \text { for } 0 \leq r \leq R_{0},
\end{array}
$$

where the coefficients $a, b, c$, and $d$ are given by

$$
\begin{gathered}
a=-\frac{\partial \mu_{A}^{0}}{\partial C_{A}}\left(C_{A}^{0}\right)-\frac{2 \eta_{0}^{2}}{\epsilon_{0}^{2}} C_{B}^{0} \\
b=\frac{\partial \mu_{B}^{0}}{\partial C_{B}}\left(C_{B}^{0}\right)+\frac{2 \eta_{0}^{2}}{\epsilon_{0}^{2}} C_{A}^{0} \\
c=1+\frac{\eta_{0}}{\epsilon_{0}} C_{B}^{0} \\
d=1-\frac{\eta_{0}}{\epsilon_{0}} C_{A}^{0},
\end{gathered}
$$

where the island shape $H_{0}$ satisfies the boundary conditions

$$
\begin{gathered}
H_{0}^{\prime}(0)=0 \\
H_{0}^{\prime}\left(R_{0}\right)=0
\end{gathered}
$$




$$
H_{0}\left(R_{0}\right)=0
$$

and from (2.27) the volume constraint becomes

$$
\int_{\mathcal{D}} H_{0} \mathrm{~d} A=1
$$

and from (2.28) the film composition $C_{B}^{1}$ satisfies

$$
\int_{V} C_{B}^{1} \mathrm{~d} V=0
$$

Note that since composition is conserved, the composition perturbation must have zero average. The remaining part of the problem is to determine $\left.T_{k k}^{S 1}\right|_{z=0}$ in terms of the island shape by solving Eqs. (3.41)-(3.44) for the axisymmetric island.

For an axisymmetric island, SS showed using Hankel transforms that the stress term in the substrate $T_{k k}^{S 1}$ is directly related to the island shape $H_{0}$ by

$$
\left.T_{k k}^{S 1}\right|_{z=0}=2(1+\nu) \mathcal{F}\left(\frac{d H_{0}}{d r}\right)
$$

where

$$
\mathcal{F}\left(\frac{d H_{0}}{d r}\right)=\int_{0}^{\infty} \xi\left[\int_{0}^{R_{0}} \hat{r} \frac{d H_{0}}{d \hat{r}} J_{1}(\xi \hat{r}) \mathrm{d} \hat{r}\right] J_{0}(\xi r) \mathrm{d} \xi .
$$

Our aim now is to find $C_{B}^{S 1}(r)$ and $H_{0}(r)$ which satisfy Eqs. (4.13)-(4.23). The island radius $R_{0}$ and the chemical potentials $\mu_{A}^{1}$ and $\mu_{B}^{1}$ are constants to be determined.

We note here a critical issue relating to the determination of $C_{B}^{1}(r, Z)$ throughout the island. At $\mathrm{O}(\delta)$, the surface equilibrium equations only prescribe

$$
C_{B}^{S 1}(r)=\left.C_{B}^{1}\right|_{Z=H_{0}(r)}
$$

To determine the composition profile within the island, we use the fact that the islands are grown slowly from zero volume. For the linearized problem, all surface quantities like $C_{B}^{S 1}$ and $H_{0}$ scale with the island size. Thus, relative to an island with the volume $V$, with shape $H_{0}$, and surface composition $C_{B}^{S 1}$, an island with volume $\frac{1}{2} V$ has shape $\frac{1}{2} H_{0}$, and surface composition $\frac{1}{2} C_{B}^{S 1}$. This similarity scaling dictates that within the island $C_{B}^{1}$ scales linearly with height and must necessarily be of the form

$$
C_{B}^{1}(r, Z)=C_{B}^{S 1}(r) \frac{Z}{H_{0}}
$$

\section{Single-Component Case}

We first show that we recover the results of SS for the single-component film. This is achieved by taking $C_{B}=0$ and $C_{A}=1$. In the limiting case $C_{B}=0$, the chemical potential of $B$ is not 
defined, so Eq. (4.14) is not relevant. Then, taking $C_{B}^{0}=0$ and $C_{B}^{1}=0$, Eq. (4.13) reduces to the one-component equation analyzed by SS,

$$
\mu_{A}^{1}=-\frac{1}{r} \frac{d}{d r}\left(r \frac{d H_{0}}{d r}\right)+\left.T_{k k}^{S 1}\right|_{z=0} \quad \text { for } 0 \leq r<R_{0}
$$

where $H_{0}$ satisfies Eqs. (4.19)-(4.22). Thus, $C_{B}=0$ gives precisely the $\mathrm{SS}$ equations for the island shape.

\section{Alloy Results}

For the general alloy problem we seek to determine $H_{0}(r)$ and $C_{B}^{S 1}(r)$ that satisfy Eqs. (4.13)(4.23), where the stresses $\left.T_{k k}^{S 1}\right|_{z=0}$ are related to the shape $H_{0}$ by Eqs. (4.24)-(4.25). We show here that combining Eqs. (4.13)-(4.14), we can reduce the problem for the shape of an alloy island to the same problem as was previously solved by SS. The surface compositions $C_{B}^{S 1}$ are then determined by the shape $H_{0}$.

We eliminate the film composition $C_{B}^{S 1}$ from Eqs. (4.13)-(4.14), to obtain

$$
\frac{b \mu_{A}^{1}-a \mu_{B}^{1}}{b c-a d}=\left.T_{k k}^{S 1}\right|_{z=0}-\frac{1}{r} \frac{d}{d r}\left(r \frac{d H_{0}}{d r}\right)
$$

for $0 \leq r<R_{0}$. Substituting Eqs. (4.24)-(4.25) into Eqs. (6.1), we obtain the integro-differential equation for the island shape $H_{0}$ :

$$
\mu_{1}=2(1+\nu) \int_{0}^{\infty} \xi\left[\int_{0}^{R_{0}} \hat{r} \frac{d H_{0}}{d \hat{r}} J_{1}(\xi \hat{r}) \mathrm{d} \hat{r}\right] J_{0}(\xi r) \mathrm{d} \xi-\frac{1}{r} \frac{d}{d r}\left(r \frac{d H_{0}}{d r}\right)
$$

for $0 \leq r<R_{0}$, where

$$
\mu_{1}=\frac{b \mu_{A}^{1}-a \mu_{B}^{1}}{b c-a d}
$$

and $H_{0}$ satisfies Eqs. (4.19)-(4.22). Eq. (6.2) is identical to the one-component problem analyzed by SS:

$$
\mu_{1}^{*}=2(1+\nu) \int_{0}^{\infty} \xi\left[\int_{0}^{R_{0}^{*}} \hat{r} \frac{d h_{0}^{*}}{d \hat{r}} J_{1}(\xi \hat{r}) \mathrm{d} \hat{r}\right] J_{0}\left(\xi r^{*}\right) \mathrm{d} \xi-\frac{1}{r^{*}} \frac{d}{d r^{*}}\left(r^{*} \frac{d h_{0}^{*}}{d r^{*}}\right)
$$

for $0 \leq r^{*}<R_{0}^{*}$, where the island shape $h_{0}^{*}$ satisfies

$$
\begin{gathered}
h_{0}^{*^{\prime}}(0)=0 \\
h_{0}^{*^{\prime}}\left(R_{0}^{*}\right)=0 \\
h_{0}^{*}\left(R_{0}^{*}\right)=0 \\
\int_{A^{*}} h_{0}^{*} \mathrm{~d} A^{*}=1,
\end{gathered}
$$


Comparing the Eqs. (6.2) and (6.4), we obtain

$$
\begin{gathered}
H_{0}=h_{0}^{*} \\
R_{0}=R_{0}^{*} \\
\frac{b \mu_{A}^{1}-a \mu_{B}^{1}}{b c-a d}=\mu_{1}^{*},
\end{gathered}
$$

where $R_{0}^{*}=1.648$ and $\mu_{1}^{*}=-0.229$ are the constants found from SS. Thus, Eqs. (6.9)-(6.10) show that the nondimensional shape of a strained alloy island is identical to the island shape for a single-component film.

To determine the composition on the island surface, we eliminate the stress term $\left.T_{k k}^{S 1}\right|_{z=0}$ from Eqs. (4.13)-(4.14) to obtain

$$
C_{B}^{S 1}(r)=\frac{d \mu_{A}^{1}-c \mu_{B}^{1}-(c-d) \frac{1}{r} \frac{d}{d r}\left(r \frac{d H_{0}}{d r}\right)}{a d-b c}
$$

for $0 \leq r<R_{0}$. Eq. (6.12) gives the film composition on the island surface in terms of the chemical potentials $\mu_{A}^{1}$ and $\mu_{B}^{1}$. To uniquely determine $\mu_{A}^{1}$ and $\mu_{B}^{1}$, we use the conservation condition Eq. (4.23). Substituting Eq. (4.27) into Eq. (4.23) and integrating by parts, we obtain

$$
d \mu_{A}^{1}-c \mu_{B}^{1}=(d-c) I
$$

where

$$
I=2 \pi \int_{0}^{R_{0}} r\left(\frac{d H_{0}}{d r}\right)^{2} \mathrm{~d} r .
$$

Eqs. (6.11) and (6.13) provide two equations from which the chemical potentials $\mu_{A}^{1}$ and $\mu_{B}^{1}$ are to be determined. The values of the chemical potentials are given by

$$
\begin{aligned}
& \mu_{A}^{1}=\frac{\left(1+\frac{\eta_{0}}{\epsilon_{0}} C_{B}^{0}\right)\left(g+\frac{2 \eta_{0}^{2}}{\epsilon_{0}^{2}}\right) \mu_{1}^{*}-\frac{\eta_{0}}{\epsilon_{0}}\left[\frac{\partial \mu_{A}^{0}}{\partial C_{A}}\left(C_{A}^{0}\right)+\frac{2 \eta_{0}^{2}}{\epsilon_{0}^{2}} C_{B}^{0}\right] I}{g+\frac{2 \eta_{0}^{2}}{\epsilon_{0}^{2}}} \\
& \mu_{B}^{1}=\frac{\left(1-\frac{\eta_{0}}{\epsilon_{0}} C_{A}^{0}\right)\left(g+\frac{2 \eta_{0}^{2}}{\epsilon_{0}^{2}}\right) \mu_{1}^{*}+\frac{\eta_{0}}{\epsilon_{0}}\left[\frac{\partial \mu_{B}^{0}}{\partial C_{B}}\left(C_{B}^{0}\right)+\frac{2 \eta_{0}^{2}}{\epsilon_{0}^{2}} C_{A}^{0}\right] I}{g+\frac{2 \eta_{0}^{2}}{\epsilon_{0}^{2}}},
\end{aligned}
$$

where

$$
g=\frac{\partial \mu_{A}^{0}}{\partial C_{A}}\left(C_{A}^{0}\right)+\frac{\partial \mu_{B}^{0}}{\partial C_{B}}\left(C_{B}^{0}\right) .
$$

Substituting Eqs. (6.15)-(6.16) into Eq. (6.12), we obtain

$$
C_{B}^{S 1}(r)=\mathcal{C}\left[I+\frac{1}{r} \frac{d}{d r}\left(r \frac{d H_{0}}{d r}\right)\right]
$$




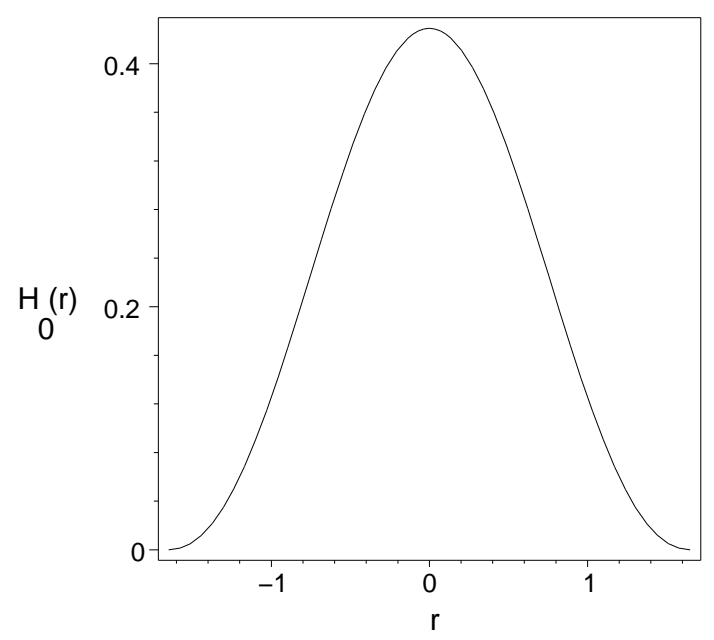

Figure 1: Island shape. Parameter value is $\nu=0.25$.

where

$$
\mathcal{C}=\frac{\frac{\eta_{0}}{\epsilon_{0}}}{g+2 \frac{\eta_{0}^{2}}{\epsilon_{0}^{2}}} .
$$

Eq. (6.18) relates the surface compositions $C_{B}^{S 1}$ to the known shape $H_{0}$, where $I$ is a known constant obtained from Eq. (6.14).

Figure1 illustrates the island shape $H_{0}(r)$, where $-R_{0} \leq r \leq R_{0}$. Figure 1 was obtained using the Bessel series solution for the island shape constructed by SS [5]. As we noticed before, the strained alloy island has the same shape as the single-component island.

The compositions on the island surface are determined by Eq. (6.18). The quantity $\mathcal{C}$ is a scale factor which determines the magnitude and the sign of the surface composition variations, while shape of the composition variations is determined by the quantity in square brackets in Eq. (6.18) which depends only on the island shape $H_{0}(r)$. Figure 2 illustrates the scaled film composition on the island surface $C_{B}^{S 1}(r) / \mathcal{C}$, where $-R_{0} \leq r \leq R_{0}$. For the given parameter values, if $\mathcal{C}>0$, $C_{B}^{S 1}(r) / \mathcal{C}$ has a negative value at $r=0$ meaning the island peak is enriched in $\mathrm{A}$ atoms, and the reduced film composition on the island surface $C_{B}^{S 1}(r) / \mathcal{C}$ attains a maximum value at two points inside of the interval $\left(-R_{0}, R_{0}\right)$ corresponding to relative enrichment of $\mathrm{B}$ atoms. For $\mathcal{C}<0$, $C_{B}^{S 1}(r) / \mathcal{C}$ has a positive value at $r=0$ meaning the island peak is enriched in $\mathrm{B}$ atoms, and the reduced film composition on the island surface $C_{B}^{S 1}(r) / \mathcal{C}$ attains a minimum value at two points inside of the interval $\left(-R_{0}, R_{0}\right)$ corresponding to relative enrichment of $\mathrm{A}$ atoms.

The magnitude of the surface composition variations is controlled by $\mathcal{C}$, which depends largely on $\eta_{0} / \epsilon_{0}$. Figure 3 shows the film composition on the island surface $C_{B}^{S 1}(r)$, where $-R_{0} \leq r \leq R_{0}$ for different values of the ratio $\eta_{0} / \epsilon_{0}$. When $\eta_{0} / \epsilon_{0}$ is positive, $C_{B}^{S 1}(r)$ has a negative value at $r=0$, which means the island peak is enriched in A atoms. When $\eta_{0} / \epsilon_{0}$ is negative, $C_{B}^{S 1}(r)$ has a positive value at $r=0$, which means the island peak is enriched in $\mathrm{B}$ atoms.

Finally, Figure 4 illustrates the reduced film composition $C_{B}^{1}(r, Z) / \mathcal{C}$ throughout the interior 


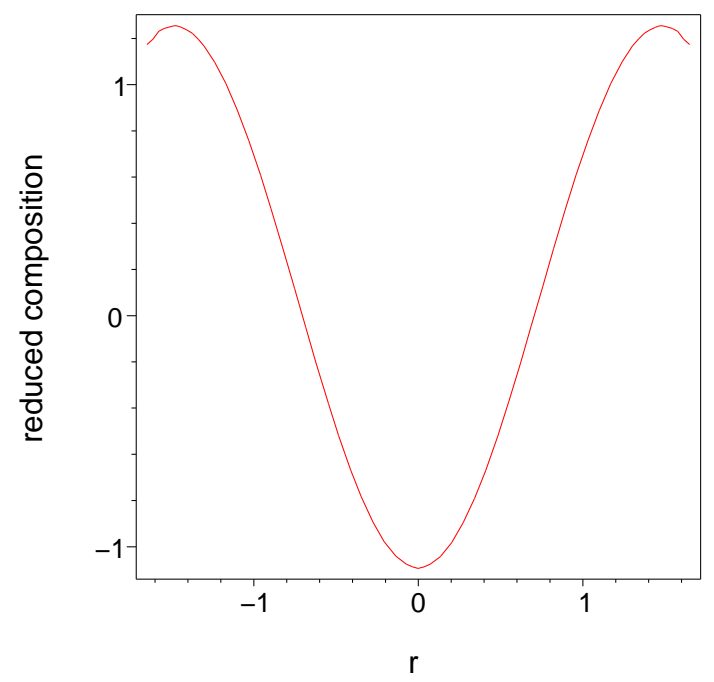

Figure 2: Reduced film composition on the island surface $C_{B}^{S 1} / \mathcal{C}$. Same island shape as in Figure 1.

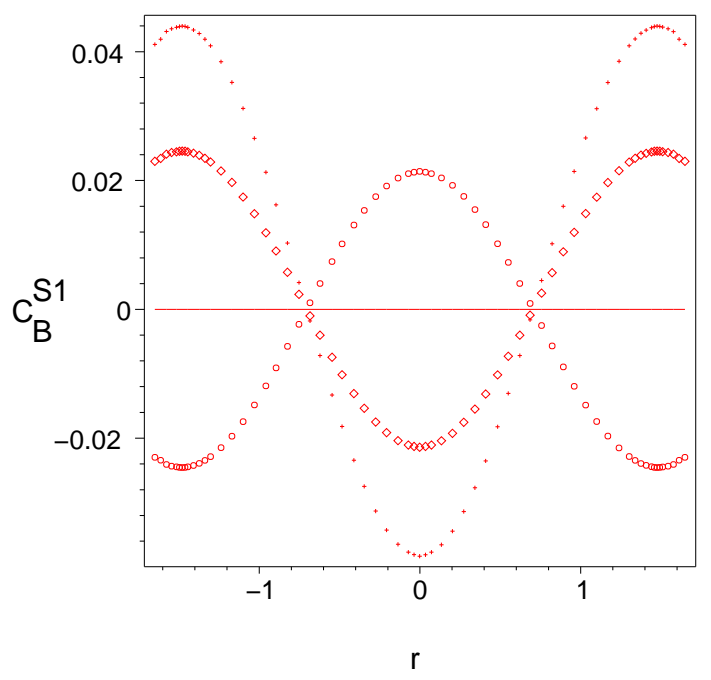

Figure 3: Film composition on the island surface $C_{B}^{S 1}$ for different values of the ratio $\eta_{0} / \epsilon_{0}$. The solid line corresponds to the case $\eta_{0} / \epsilon_{0}=0$. The curves represented by the symbols circle, diamond, and cross correspond to $\eta_{0} / \epsilon_{0}=-1, \eta_{0} / \epsilon_{0}=1$, and $\eta_{0} / \epsilon_{0}=2$, respectively. Other parameters values are $C_{B}^{0}=0.5, \nu=0.25, \Omega=8.66, T=16.60$. 


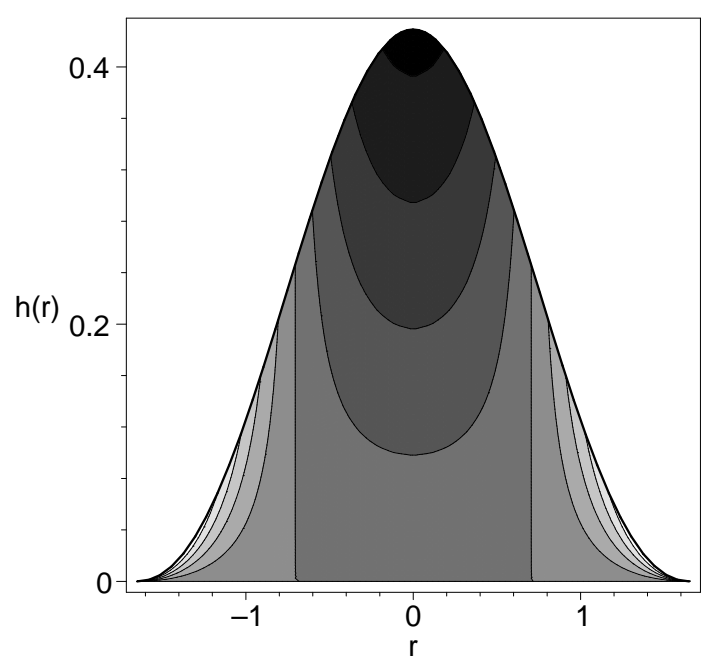

Figure 4: Contour plot of reduced film composition $C_{B}^{1} / \mathcal{C}$. Step between contours is 0.25 with dark regions corresponding to negative values. Vertical lines are the zero contour. Other parameters values are $C_{B}^{0}=0.5, \eta_{0}=4, \epsilon_{0}=2, \nu=0.25, \Omega=8.66, T=16.60$.

of the island, where $-R_{0} \leq r \leq R_{0}$ and $0 \leq Z \leq H_{0}(r)$. If $\mathcal{C}>0$, then $C_{B}^{1}$ is minimum near the island peak and maximum near the surface close to the edges of the island. For $\mathcal{C}<0$, the maximum/minimum locations are reversed.

\section{Results for $S \mathbf{i}_{1-X} \mathbf{G e}_{X}$ on $\mathbf{S i}_{1-Y} \mathbf{G e}_{Y}$}

To illustrate further our theoretical results, we make a direct comparison to SiGe film growth, where the film and substrate are of the same alloy but of different compositions: $\mathrm{Si}_{1-X} \mathrm{Ge}_{X}$ films on $\mathrm{Si}_{1-Y} \mathrm{Ge}_{Y}$ substrates. Thus, the misfit strain of the film can be expressed in terms of the atomic size difference and the composition difference in the film and substrate as $\epsilon_{0}=\eta_{0}(Y-X)$. For comparison, we take $\eta_{0}=4, \nu=0.25, \Omega=8.66$, and $T=16.60$ as typical parameter values for SiGe alloys.

Figure 5 shows how the Ge composition on the island peak, $C_{B}^{S 1}(0)$, depends on the Ge film composition $X$. On the diagonal, since $X=Y$ there is no misfit $\epsilon_{0}$, and thus there is no composition variation. The maximum/minimum composition attained at the peak is controlled by $\mathcal{C}$, which depends on $\eta_{0}, \epsilon_{0}$, and $X$. Figure 5 illustrates that maximum/minimum peak values are obtained for regions of $(X, Y)$ lying off the diagonal.

Finally, we compare our theory for small "prepyramid" islands to recent experiments $[4,1]$ which measure the composition profile in a large "pyramid" or "dome" islands. In Denker et al [1], intermixed SiGe pyramid islands were observed on $\mathrm{Si}$ and it was found that the pyramid peak was enriched in Ge relative to the rest of the island. In Malachias et al [4], intermixed SiGe domes on Si substrates were observed, and their composition map showed that the dome peak was 


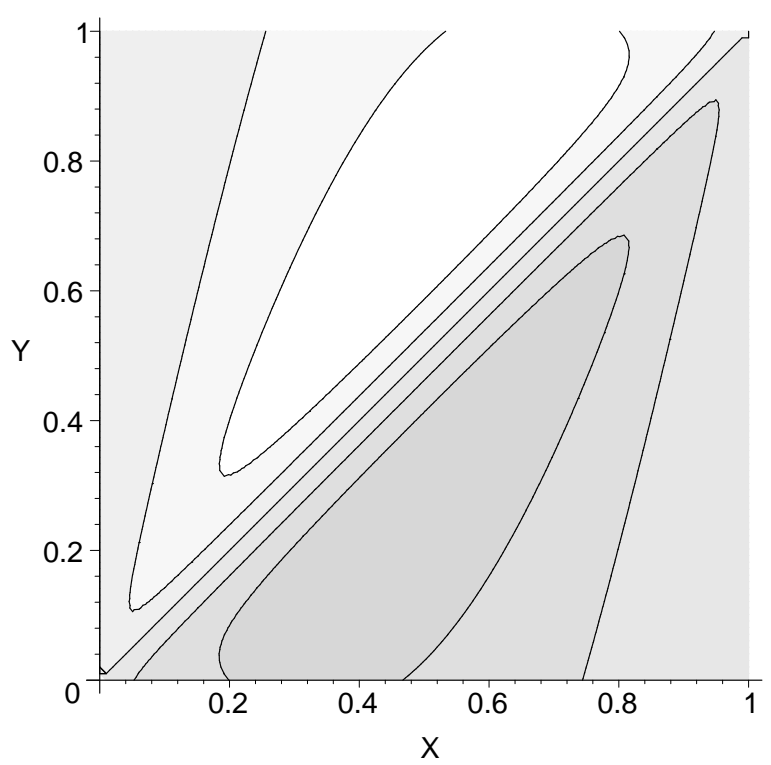

Figure 5: Ge composition on the island peak $C_{B}^{S 1}(0)$. Step between contours is 0.02 with dark regions corresponding to positive values. Zero contour is diagonal line. Other parameters values are $\eta_{0}=4, \nu=0.25, \Omega=8.66, T=16.60$.

enriched in Ge. Since our theory is valid only for small islands, we do not expect quantitative agreement, but our theory does give good qualitative agreement for the segregation pattern in the island. An intermixed GeSi island on a Si substrate lies along the $Y=0$ axis of Figure 5, with all alloy film compositions $X$ with $0<X<1$ having a composition perturbation at the island peak, $C_{B}^{S 1}(0)$ enriched in Ge.

Comparison of the small-island composition map, Figure 2, to the experiments shows some similarity for pyramids, but not for domes. In the pyramids of Denker et al the Ge rich region forms an "inverted pyramid" structure which is roughly similar in shape to the "inverted cone" structure of the Ge-rich region in Figure 2. In the larger dome islands observed by Malachias et al, however, the shape of the Ge-rich region is more of a shell-like structure in which the isocomposition contours mimic the shape of the dome, which is clearly different from that suggested in the small-volume limit for the prepyramid island in Figure 2. So we do not suggest that our theory can be extrapolated to large volume islands.

We attempt to quantify the magnitude of the composition variations from our theory and how these compare to the observations in the larger dome island. Our small-amplitude theory gives a linear variation in the composition with depth, as in Eq. (4.27). Thus, the composition along a vertical line through the center of the island is given in dimensional form by

$$
C_{B}=C_{B}^{0}+\frac{V^{*}}{l^{3}} C_{B}^{S 1}(0) \frac{z^{*}}{h^{*}}
$$

where $V^{*}$ and $h^{*}$ are the dimensional island volume and height, $z^{*}$ is the (dimensional) vertical coordinate, and $l=\gamma(1-\nu) / \epsilon_{0}^{2} E$ is the length scale. In Malachias et al, the dome is about $15 \mathrm{~nm}$ 
tall and $60 \mathrm{~nm}$ in diameter with composition ranging from $0 \% \mathrm{Ge}$ at the island base to $100 \%$ at the island peak. Since the composition range is saturated, there clearly must be nonlinear effects playing a role, but it is instructive to see what type of composition variation is generated within the small-island theory. If we approximate the dome as a spherical cap, it has volume $V^{*}=2 \times 10^{4} \mathrm{~nm}^{3}$, and using GeSi materials parameters for $X=0.5\left(\gamma=2220 \mathrm{erg} / \mathrm{cm}^{2}, E=11.6 \times 10^{11} \mathrm{erg} / \mathrm{cm}^{3}\right.$, $\nu=0.276, \epsilon_{0}=0.021$ ) we obtain a length scale $l=31.4 \mathrm{~nm}$. For a GeSi island with composition $X=0.5$, Figure 5 gives $C_{B}^{S 1}(0)=0.04$. Thus our composition profile along the island centerline would be

$$
C_{B} \approx C_{B}^{0}+0.05 \frac{z^{*}}{h^{*}} \text {. }
$$

Thus, for an island with the size of the large dome in Malachias et al, our theory would predict that the composition ranged from about $C_{B}=0.5$ at the island base to about $C_{B}=0.55$ at the island peak, which is substantially less than what is observed. So, as with the shape of the composition variation in the island, our theory does not extrapolate well to large islands, which is not surprising since for the dome, $\delta=V^{*} / l^{3}=1.35$ which is not small. A separate issue, as suggested in [6], is that our small island theory specifically rules out diffusional intermixing with the substrate which may be an important contributor to the composition structure at high temperatures and/or slow growth rates.

\section{Conclusion}

In this paper we have analyzed one aspect of morphology development relevant to alloy film growth: island formation. We have considered a detailed model for strained film growth, which describes the moving boundary with underlying composition and strain, and the coupling between strain, composition and the morphology of the free boundary. We have analyzed the equilibrium morphology of a strained AB alloy film for the case where it wets the substrate (Stranski-Krastanow growth). We have found the shape and the composition profile of an axisymmetric island considering an asymptotic solution based on the island height being much smaller that the island width. By mapping the alloy problem to the single-component problem previously solved by Shanahan and Spencer [5], we determined that the nondimensional shape of a strained alloy island is identical to the island shape for a single-component film (in the limit of small island size). From the island shape, we then determined the composition profile everywhere in the island and identified a key parameter controlling the magnitude of compositional uniformity.

For the case of $\mathrm{Si}_{1-X} \mathrm{Ge}_{X}$ films on $\mathrm{Si}_{1-Y} \mathrm{Ge}_{Y}$ substrates, we have described how the Ge composition on the island peak depends on the film and substrate compositions. We have compared our theoretical results to recent experiments measuring the composition profile in large "dome" islands. Although our theory is valid only for small islands, we have obtained qualitative agreement of our theory with these experiments in which there is a Ge rich region near the island peak and decreasing Ge regions moving vertically downwards closer to the substrate interface. 


\section{Acknowledgements}

This research was supported by National Science Foundation through a Nanoscale Interdisciplinary Research Team grant DMR-0102794, and DMS-0505497.

\section{References}

[1] U. Denker, M. Stoffel, O. G. Schmidt. Probing the lateral composition profile of selfassembled islands. Phys. Rev. Lett., 90 (2003), 196102.

[2] H. Gao. Some general properties of stress-driven surface evolution in heteroepitaxial thin film structure. J. Mech. Phys. Solids, 42 (1994), 741-772.

[3] H. Gao, W. D. Nix. Surface roughening of heteroepitaxial thin films. Annu. Rev. Mater. Sci., 29 (1999), 173-209.

[4] A. Malachias, S. Kycia, G. Medeiros-Ribeiro, R. Magalhães-Paniago, T. I. Kamins, R. S. Williams. 3D composition of epitaxial nanocrystals by anomalous X-ray diffraction: observation of a Si-rich core in Ge domes on Si(100). Phys. Rev. Lett., 91 (2003), 176101.

[5] L. L. Shanahan, B. J. Spencer. A codimension-two free boundary problem for the equilibrium shapes of a small three-dimensional island in an epitaxially strained solid film. Interfaces and Free Boundaries, 4 (2002), 1-25.

[6] V. A. Shchukin, D. Bimberg. Spontaneous ordering of nanostructures on crystal surfaces. Rev. Mod. Phys., 71 (1999), 1125-1171.

[7] B. J. Spencer. An asymptotic derivation of the glued wetting layer model and contact angle condition for Stranski-Krastanow islands. Phys. Rev. B, 59 (1999), 2011-2017.

[8] B. J. Spencer, M. Blanariu. Shape and composition profile of a prepyramid quantum dot. Phys. Rev. Lett., 95 (2005), 206101.

[9] B. J. Spencer, P. W. Voorhees, J. Tersoff. Morphological instability theory for strained alloy film growth: the effect of compositional stresses and species-dependent surface mobilities on ripple formation during epitaxial film deposition. Phys. Rev. B, 64 (2001), 235318. 